Questions vives

\section{Questions Vives}

Recherches en éducation

$n^{\circ} 21 \mid 2014$

Le travail collectif des enseignants en question(s)

\title{
Bailleul, M., Thémines, J.-F., \& Wittorski, R. (Coordinateurs). Expériences et développement professionnel des enseignants : formation, travail, itinéraire professionnel
}

Toulouse : Octares Éditions

Javier Nunez Moscoso

\section{(2) OpenEdition Journals}

\section{Édition électronique}

URL : http://journals.openedition.org/questionsvives/1500

DOI : 10.4000/questionsvives. 1500

ISSN : 1775-433X

Éditeur

Université Aix-Marseille (AMU)

\section{Édition imprimée}

Date de publication : 15 septembre 2014

ISBN : 978-2-912643-45-2

ISSN : 1635-4079

\section{Référence électronique}

Javier Nunez Moscoso, «Bailleul, M., Thémines, J.-F., \& Wittorski, R. (Coordinateurs). Expériences et développement professionnel des enseignants : formation, travail, itinéraire professionnel », Questions Vives [En ligne], $n^{\circ} 21$ | 2014, mis en ligne le 15 septembre 2014, consulté le 22 septembre 2020. URL http://journals.openedition.org/questionsvives/1500; DOI : https://doi.org/10.4000/questionsvives. 1500

Ce document a été généré automatiquement le 22 septembre 2020.

\section{c)}

Questions Vives est mis à disposition selon les termes de la licence Creative Commons Attribution Pas d'Utilisation Commerciale - Pas de Modification 4.0 International. 
Bailleul, M., Thémines, J.-F., \& Wittorski, R. (Coordinateurs). Expériences et développement professionnel des enseignants : formation, travail, itinéraire professionnel

\author{
Toulouse : Octares Éditions
}

Javier Nunez Moscoso

\title{
RÉFÉRENCE
}

Bailleul, M., Thémines, J.-F., \& Wittorski, R. (Coordinateurs). Expériences et développement professionnel des enseignants : formation, travail, itinéraire professionnel. Toulouse : Octares Éditions.

1 Cet ouvrage collectif formalise une réflexion initiée à l'occasion du colloque «Recherche(s) en éducation et en formation. Développement professionnel des enseignants " (Rouen, 3-5 juin 2009). Les diverses contributions se proposent, d'une part, de "comprendre le développement professionnel des enseignants, à partir de l'étude des expériences de formation, de travail et des itinéraires professionnels des individus » (p.1) et, d'autre part, d'analyser comment le développement professionnel a été investi par la recherche, dans l'étude des pratiques enseignantes et dans le champ de la formation.

2 Pour ce faire, l'écrit est divisé en trois parties. La première partie, intitulée « Éclairages sur le développement professionnel » convoque des regards pluriels (mais revendiqués comme étant complémentaires) sur le lien entre formation et développement 
professionnel. Dans le premier des trois chapitres qui la composent, Jean-Marie Barbier explique l'importance et l'ampleur de la professionnalisation et du développement professionnel dans l'actualité du travail et de la formation. Le deuxième chapitre, de la plume de Françoise Cros, aborde les liens entre l'écriture (autant en recherche comme dans le champ professionnel) et le développement professionnel des acteurs et leurs apports mutuels. Le troisième chapitre, sous la signature d'Anne Jorro, explore la question de comment l'évaluation peut être pensée comme source de développement professionnel.

3 La deuxième partie du livre, appelée « Apprendre le métier : conceptions, compétences, identités", étudie le développement professionnel engagé dans la formation des enseignants et, plus spécifiquement, durant l'année de stage et la première année d'exercice professionnel des enseignants. Elle rend compte d'un projet conduit dans deux académies, ayant pour objectif l'étude de la professionnalisation au cours du processus de titularisation, autant dans les espaces formels de formation que dans les situations de travail. Nous retrouvons deux démarches dans cette partie, une quantitative (4 chapitres) et une qualitative (1 chapitre).

Dans le chapitre quatre, Richard Wittorski et Marc Bailleul présentent les aspects théoriques et méthodologiques de l'enquête longitudinale. Par une approche alimentée à la fois par la sociologie des professions anglo-saxonne et par l'ergonomie et la psychologie du travail, les auteurs proposent de séparer ce qui relève de la professionnalisation (émanant de l'intention sociale ou institutionnelle ou de l'injonction de provoquer un changement dans le travail des individus) de ce qui relève du développement professionnel (mouvement conjoint entre l'activité, les stratégies et les dynamiques identitaires, c'est-à-dire des éléments impliqués dans le processus de construction ou développement de l'individu vis-à-vis de sa profession). L'hypothèse de l'existence de stratégies identitaires mises en œuvre par les enseignants est développée par les trois chapitres suivants : Bodergat et Themines (chapitre 5) qui s'intéressent à l'évolution des significations de l'activité chez les enseignants entre l'année de stage et la première année d'enseignement à proprement parler ; Tavignot et Janner (chapitre 6) qui s'interrogent sur les compétences professionnelles perçues comme construites par les enseignants, ainsi que les différences entre l'année de stage et l'année de titularisation à partir de celles repérées dans le cahier de charges de la formation en 2006 ; et Buhot et Cosnefroy (chapitre 7) qui abordent les différentes modalités de construction de compétences professionnelles, où la place du terrain et notamment des acteurs, semble première.

5 La deuxième démarche de type qualitatif est représentée par le chapitre de Zimmermann, Meard et Flavier (chapitre 8). En se questionnant sur l'existence d'une identité professionnelle spécifique aux enseignants débutants, les auteurs proposent une entrée par la psychologie historico-culturelle et par la clinique de l'activité. Ce travail qui clôt la deuxième partie de l'ouvrage défend l'idée d'une identité en construction permanente, en proposant l'hypothèse que la seule spécificité des débutants semble être la préoccupation immédiate pour les «mobiles » (surmonter les difficultés quotidiennes, par exemple les moments de malaise), en minorant les opérations pour apprendre le métier de caractère plus stable (comme les rôles).

6 La troisième partie de ce livre collectif s'intitule « Discontinuités : le rôle des situations et des contextes dans le développement professionnel». Elle est articulée en 6 chapitres qui possèdent l'ambition commune d'étudier la contribution des ruptures 
perçues par les acteurs (dans leur travail et leur trajectoire) à leur développement professionnel.

7 Luc Ria (chapitre 9) tente de préciser la nature des "passages à risques" des enseignants novices travaillant dans des milieux difficiles. Il envisage les moments critiques du travail vécus par l'acteur comme sollicitant énormément d'émotions et d'énergie. À travers des témoignages, l'auteur décrit une partie du processus de tâtonnement des enseignants débutants pour trouver des stratégies de pilotage de la classe. Il présente aussi quelques dispositifs d'accompagnement mis en place par certains établissements pour analyser les pratiques des débutants.

Dans le chapitre 10, Christine Félix et Frédéric Saujat problématisent les nouvelles dynamiques organisationnelles issues de l'importante incorporation d'enseignants expérimentés et d'assistants pédagogiques dans les 249 Réseaux Ambition Réussite (RAR), en s'interrogeant sur le développement professionnel attaché à ces processus de recomposition du travail et aux changements dans les pratiques des partenaires. Ce travail souligne l'absence de prescriptions claires qui sont sources de contraintes et pointe la difficulté majeure du travail collectif entre enseignants et assistants pédagogiques: les tensions dans «l'articulation de l'usage de soi par soi, de l'usage de soi par l'autre et de l'usage de l'autre par soi » (p. 136).

9 Le chapitre 11 est cosigné par Marie-Estelle Rouve et Luc Ria, qui étudient le lien entre la transformation des contextes de travail dans les classes du RAR et le processus de construction de l'activité chez les enseignants novices, à partir d'une entrée par l'identité professionnelle. En mobilisant le cadre du cours d'action, l'étude montre l'impact positif ou négatif des collectifs de travail dans la construction de l'identité professionnelle, en mettant en avant l'hétérogénéité des contextes (élèves, parents, enseignants, équipes, etc.). La stabilisation des normes d'action chez les enseignants se fait progressivement, en lien avec les retours des collègues et la mise en contraste de leurs façons de faire.

10 Xavière Laneelle, dans le chapitre 12, aborde le cas des enseignants vacataires, en se questionnant par les effets des conditions de travail vis-à-vis de leur développement professionnel (phases d'activité et d'inactivité professionnelle, suppléance des absences face à des classes différentes, budget provisoire, etc.). À partir d'une inspiration libre de la sociologie des réseaux et de l'analyse configurationnelle, les résultats révèlent que «l'intermittence " professionnelle à laquelle ils sont soumis, restreint fortement le développement professionnel à la fois par les conditions de travail et par l'accueil dans les établissements, au travers de la réduction des espaces de collaboration et partage.

11 La recherche de Thérèse Perez-Roux (chapitre 13) porte sur le processus de construction identitaire chez les enseignants stagiaires en lycée professionnel. Dans l'objectif de comprendre l'influence des trajectoires professionnelles, des situations de travail et de contextes de formation en l'identité professionnelle, elle montre que le passage d'un métier à un autre, provoque le basculement des représentations préalables et l'élaboration de nouvelles, changement nécessaire pour « donner sens » à l'activité.

12 Le chapitre 14, écrit par Anne-Laure Le Guern, est le dernier de l'ouvrage. Celui-ci défend l'idée que le développement professionnel n'est possible qu'à condition de surmonter les épreuves et les défis du travail. Ce processus engage des prises de 
conscience, des changements identitaires et des manières de penser et de faire, ainsi qu'une certaine identification et le partage des difficultés au travail.

L'ouvrage se termine par une conclusion générale qui met en exergue la tension entre la logique du développement professionnel, davantage centré sur le sujet, et la logique de la professionnalisation, centrée sur les dispositifs institutionnels. Le principal apport de cet ouvrage est d'explorer certaines articulations entre ces deux points de vue opposés, au travers, en particulier, d'une complexification de l'objet d'étude, réfutant simultanément une explication du développement par la professionnalisation et l'inverse.

14 Nous retiendrons également l'idée force selon laquelle le développement professionnel a lieu dans la réflexion des acteurs sur les épisodes critiques de l'activité, tout en étant lié aux itinéraires et aux contextes d'action des enseignants.

\section{AUTEURS}

\section{JAVIER NUNEZ MOSCOSO}

Chercheur associé UMR-EFTS, Université de Toulouse 